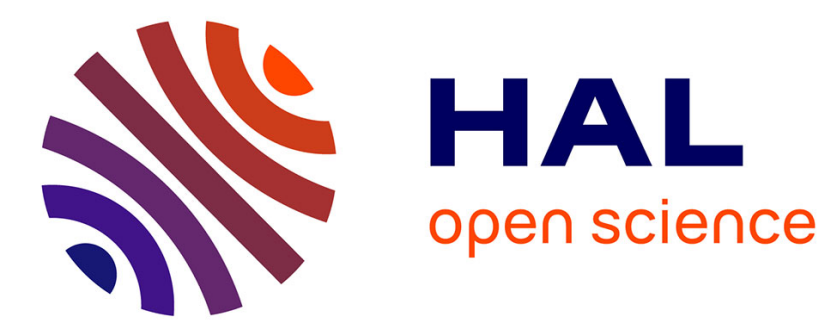

\title{
Extreme paths in oriented two-dimensional percolation
}

\author{
E. D. Andjel, L. F. Gray
}

\section{To cite this version:}

E. D. Andjel, L. F. Gray. Extreme paths in oriented two-dimensional percolation. Journal of Applied Probability, 2016, 53 (02), pp.369-380. 10.1017/jpr.2016.6 . hal-01302642

\section{HAL Id: hal-01302642 \\ https://hal.science/hal-01302642}

Submitted on 14 Apr 2016

HAL is a multi-disciplinary open access archive for the deposit and dissemination of scientific research documents, whether they are published or not. The documents may come from teaching and research institutions in France or abroad, or from public or private research centers.
L'archive ouverte pluridisciplinaire HAL, est destinée au dépôt et à la diffusion de documents scientifiques de niveau recherche, publiés ou non, émanant des établissements d'enseignement et de recherche français ou étrangers, des laboratoires publics ou privés. 


\title{
EXTREME PATHS IN ORIENTED 2D PERCOLATION
}

\author{
E. D. ANDJEL, ${ }^{*}$ Université d'Aix-Marseille \\ L. F. GRAY, ${ }^{* *}$ University of Minnesota
}

\begin{abstract}
A useful result about leftmost and rightmost paths in two dimensional bond percolation is proved. This result was introduced without proof in [5] in the context of the contact process in continuous time. As discussed here, it also holds for several related models, including the discrete time contact process and two dimensional site percolation. Among the consequences are a natural monotonicity in the probability of percolation between different sites and a somewhat counter-intuitive correlation inequality.

Keywords: Oriented percolation; extreme paths; inequalities.

2010 Mathematics Subject Classification: Primary 60K35
\end{abstract}

Secondary 60J10

\section{Introduction}

The main goal of this paper is to give complete proofs of a result originally presented in [5]. The result was stated for the continuous time contact process in [5], but its proof is missing in the literature. In that paper some interesting consequences are given which we believe justify the writing of the proof here. In the present paper we work in the context of oriented two dimensional percolation which is equivalent to a discrete time version of the contact process. In the latter part of this paper we discuss how our results apply to other models, and derive some consequences following the ideas of [5].

Two dimensional oriented bond percolation is studied in [4], where some of its most

\footnotetext{
* Postal address: 39 Rue Joliot Curie,13453 Marseille, France. Email address: andjel@impa.br ** Postal address: School of Mathematics, Minneapolis MN 55455-0488. Email address: gray@math.umn.edu
} 
important properties are proved. To introduce the model, let

$$
\Lambda=\{(x, y): x, y \in \mathbb{Z}, y \geq 0, x+y \in 2 \mathbb{Z}\}
$$

Then, draw oriented edges from each point $(m, n)$ in $\Lambda$ to $(m+1, n+1)$ and to $(m-1, n+1)$. In the percolation literature, the points in $\Lambda$ and the edges between them are often called sites and bonds, respectively. In this paper we focus on oriented bond percolation, and thus we suppose that the edges are open independently of each other, and that each edge is open with probability $p \in(0,1)$.

It is an easy matter to adapt the proof here to oriented site percolation, in which the points, rather than the edges, are open with probability $p$, independently of each other. And either by using the so-called "graphical construction" of continuous time interacting particle systems, or by taking limits of discrete time contact processes, our methods can also be applied to various versions of the continuous time contact process in one dimension. For more about such extensions, see our discussion in Section 3.

A path $\pi$ in $\Lambda$ is a sequence $\left(x_{0}, y_{0}\right), \ldots,\left(x_{n}, y_{n}\right)$ of points in $\Lambda$ such that for all $0 \leq i<n,\left|x_{i+1}-x_{i}\right|=1$ and $y_{i+1}-y_{i}=1$. The edges joining $\left(x_{i}, y_{i}\right)$ to $\left(x_{i+1}, y_{i+1}\right)$ for $0 \leq i \leq n-1$ will be called the edges of $\pi$. We say that a path is open if all its edges are open. (In site percolation, a path is open if all its points are open.)

For any $n \in \mathbb{N}_{0}$ let $L_{n}=\{(x, n) \in \Lambda\}$. Let $0 \leq m<n$ and let $A$ and $B$ be subsets of $L_{m}$ and $L_{n}$ respectively. A path from $A$ to $B$ is any path starting in some point in $A$ and finishing at some point in $B$. A path $\pi$ from a point in $L_{m}$ to a point in $L_{n}$ will be identified with the function $\pi:[m, n] \cap \mathbb{Z} \rightarrow \mathbb{Z}$ determined by: $(\pi(j), j)$ is a point in the path $\pi$ for all $m \leq j \leq n$.

Given two paths $\pi_{1}$ and $\pi_{2}$ from $L_{m}$ to $L_{n}$ we say that $\pi_{1}$ is to the left of $\pi_{2}$ (or that $\pi_{2}$ is to the right of $\pi_{1}$ ) and write $\pi_{1} \leq \pi_{2}$ (or $\pi_{2} \geq \pi_{1}$ ) if $\pi_{1}(j) \leq \pi_{2}(j)$ for all $m \leq j \leq n$. This creates a partial order between paths from $L_{m}$ to $L_{n}$. If the inequality is replaced by strict inequality, then we say that $\pi_{1}$ is strictly to the left of $\pi_{2}$ (or $\pi_{2}$ is strictly to the right of $\pi_{1}$ ).

We find it useful to extend the notions "strictly to the left" and "strictly to the right" to subsets of $\Lambda$. Let $P_{1}: \Lambda \rightarrow \mathbb{Z}$ be the projection on the first coordinate: $P_{1}((x, y))=x$. And in the usual fashion, extend this function to sets $\Lambda^{\prime} \subset \Lambda: P\left(\Lambda^{\prime}\right)=$ $\left\{P_{1}((x, y)):(x, y) \in \Lambda^{\prime}\right\}$. For $G$ a subset of $\Lambda$, we denote by $\ell(G)$ the set of all points 
$(j, k) \in \Lambda$ such that $j<\inf P_{1}\left(G \cap L_{k}\right)$, and we denote by $r(G)$ the set of points $(j, k) \in \Lambda$ such that $j>\sup P_{1}\left(G \cap L_{k}\right)$. (Here by convention, $\sup \emptyset=-\infty$ and $\inf \emptyset=\infty$.) Thus, $\ell(G)(r(G))$ is the set of all points in $\Lambda$ that are strictly to the left (right) of $G$. If $G, G^{\prime}$ are subsets of $\Lambda$, we say that $G$ is strictly to the left of $G^{\prime}$ or, equivalently, $G^{\prime}$ is strictly to the right of $G$, if $G \subset \ell\left(G^{\prime}\right)$, or equivalently if $G^{\prime} \subset r(G)$. And if $\pi$ is a path, then we say that $\pi$ is strictly to the left of $G$ (strictly to the right of $G$ ) if the set of points in $\pi$ is strictly to the left of $G$ (strictly to the right of $G$ ). The notation $\ell(\cdot)$ and $r(\cdot)$ introduced here also applies to paths, thinking of them as sets. For example, a path $\pi$ is strictly to the left of a set $G$ if and only if $G \subset r(\pi)$. Please note that this terminology and notation are consistent with our earlier definition of one path being strictly to the left of another path, but that they now also apply to paths that do not necessarily start on the same level $L_{m}$ or end on the same level $L_{n}$.

We also extend these notions to edges: we say that an edge $e$ is to the left (right) of a path $\gamma$ if each of the endpoints of $e$ is either on $\gamma$ or strictly to the left (right) of $\gamma$. If in addition, at least one of these endpoints is strictly to the left (right) of $\gamma$, we say that $e$ is strictly to the left (right) of $\gamma$. Note that this differs slightly from our definitions of "strictly to the left" for paths or sets, because we do not require the whole edge to be strictly to the left of a path and one of its endpoints may belong to the path.

Let $\Lambda^{\prime} \subset \Lambda$. Note that, if $A \subset L_{m}$ and $B \subset L_{n}$ are finite and there is at least one path from $A$ to $B$ contained in $\Lambda^{\prime}$, then there is a unique path from $A$ to $B$ contained in $\Lambda^{\prime}$ which is to the left of all paths from $A$ to $B$ contained in $\Lambda^{\prime}$. This is called the leftmost path from $A$ to $B$ in $\Lambda^{\prime}$. And, if there is an open path from $A$ to $B$ contained in $\Lambda^{\prime}$, then there is a unique open path from $A$ to $B$ contained in $\Lambda^{\prime}$ which is to the left of all open paths from $A$ to $B$ contained in $\Lambda^{\prime}$. This path will be called the leftmost open path from $A$ to $B$ in $\Lambda^{\prime}$. Similarly, we define the rightmost path and rightmost open path from $A$ to $B$ in $\Lambda^{\prime}$.

Given a subset $\Lambda^{\prime}$ of $\Lambda, 0 \leq m<n \in \mathbb{N}$ and two finite subsets $A$ and $B$ of $L_{m}$ and $L_{n}$ respectively, $\Gamma_{\Lambda^{\prime}}(A, B)$ will denote the set of paths from $A$ to $B$ contained in $\Lambda^{\prime}$. If this set is non-empty, then $\mu_{\Lambda^{\prime}}(A, B)\left(\nu_{\Lambda^{\prime}}(A, B)\right)$ will denote the conditional distribution of the leftmost (rightmost) open path from $A$ to $B$ contained in $\Lambda^{\prime}$ given that there is at least one open path from $A$ to $B$ in $\Lambda^{\prime}$. In all these notations the 
subscript $\Lambda^{\prime}$ will be omitted if $\Lambda^{\prime}$ is the whole set $\Lambda$, and when either $A$ or $B$ is a singleton, say $\{(x, y)\}$, we will often write $(x, y)$ rather than $\{(x, y)\}$.

Let $0 \leq m<n$, let $A$ and $B$ be finite subsets of $L_{m}$ and $L_{n}$ respectively such that $\Gamma(A, B)$ is nonempty, and let $\mu$ and $\nu$ be probability measures on $\Gamma(A, B)$. We say that $\mu$ is stochastically to the left of $\nu$ and write $\mu \leq \nu$ if for any increasing function $\Phi$ on $\Gamma(A, B)$ we have

$$
\int_{\Gamma(A, B)} \Phi(\gamma) d \mu(\gamma) \leq \int_{\Gamma(A, B)} \Phi(\gamma) d \nu(\gamma)
$$

We can now state our version of the main result in [5]:

Theorem 1. Let $0 \leq m<n$, let $A$ and $B$ be finite subsets of $L_{m}$ and $L_{n}$ respectively and let $\Lambda^{\prime}$ and $G$ be subsets of $\Lambda$. If $\Gamma_{\Lambda^{\prime} \cap \ell(G)}(A, B)$ is nonempty, we have $\mu_{\Lambda^{\prime} \cap \ell(G)}(A, B) \leq \mu_{\Lambda^{\prime}}(A, B)$ and $\nu_{\Lambda^{\prime} \cap \ell(G)}(A, B) \leq \nu_{\Lambda^{\prime}}(A, B)$. And if $\Gamma_{\Lambda^{\prime} \cap r(G)}(A, B)$ is nonempty, we have $\mu_{\Lambda^{\prime} \cap r(G)}(A, B) \geq \mu_{\Lambda^{\prime}}(A, B)$ and $\nu_{\Lambda^{\prime} \cap r(G)}(A, B) \geq \nu_{\Lambda^{\prime}}(A, B)$.

This result has the following immediate corollary:

Corollary 1. Let $m<n$, let $\Lambda^{\prime}$ be a subset of $\Lambda$, let $A$ and $B$ be finite subsets of $L_{m}$ and $L_{n}$ respectively such that $\Gamma_{\Lambda^{\prime}}(A, B)$ is nonempty and suppose $A^{\prime}$ is a finite subset of $L_{m}$ that is strictly to the right of $A$. Then, $\mu_{\Lambda^{\prime}}\left(A \cup A^{\prime}, B\right) \geq \mu_{\Lambda^{\prime}}(A, B)$ and $\nu_{\Lambda^{\prime}}\left(A \cup A^{\prime}, B\right) \geq \nu_{\Lambda^{\prime}}(A, B)$. Moreover, if $B^{\prime}$ is a finite subset of $L_{n}$ that is strictly to the right of $B$, then $\mu_{\Lambda^{\prime}}\left(A, B \cup B^{\prime}\right) \geq \mu_{\Lambda^{\prime}}(A, B)$ and $\nu_{\Lambda^{\prime}}\left(A, B \cup B^{\prime}\right) \geq \nu_{\Lambda^{\prime}}(A, B)$. If, instead $A^{\prime}$ is strictly to the left of $A$ ( $B^{\prime}$ is strictly to the left of $B$ ) then the first two (last two) inequalities are reversed.

The different parts of the corollary follow from the theorem by making appropriate choices of the sets $A, B, G$. For example, for the first part of the corollary, replace $A$ in the theorem by $A \cup A^{\prime}$ and let $G=A^{\prime}$. As an immediate consequence of this corollary we get:

Corollary 2. Assume that $m, n, \Lambda^{\prime}, A$ and $B$ are as in Corollary 1. If $A^{\prime}$ is a subset of $L_{m}$ strictly to the right of $A$ and $\Gamma_{\Lambda^{\prime}}\left(A^{\prime}, B\right)$ is nonempty, then $\mu_{\Lambda^{\prime}}\left(A^{\prime}, B\right) \geq \mu_{\Lambda^{\prime}}(A, B)$ and $\nu_{\Lambda^{\prime}}\left(A^{\prime}, B\right) \geq \nu_{\Lambda^{\prime}}(A, B)$. Similarly, if $B^{\prime}$ is a finite subset of $L_{n}$ strictly to the right of $B$ and $\Gamma_{\Lambda^{\prime}}\left(A, B^{\prime}\right)$ is nonempty, then $\mu_{\Lambda^{\prime}}\left(A, B^{\prime}\right) \geq \mu_{\Lambda^{\prime}}(A, B)$ and $\nu_{\Lambda^{\prime}}\left(A, B^{\prime}\right) \geq$ $\nu_{\Lambda^{\prime}}(A, B)$. 
Each of the four inequalities of this corollary is obtained applying twice Corollary 1. For example to obtain the first of these inequalities write: $\mu_{\Lambda^{\prime}}\left(A^{\prime}, B\right) \geq \mu_{\Lambda^{\prime}}\left(A \cup A^{\prime}, B\right) \geq$ $\mu_{\Lambda^{\prime}}(A, B)$.

In Section 2, we give a proof of our main theorem, based on a Markov chain that was introduced in [3]. In that paper, the Markov Chain was used to prove that the one dimensional nearest neighbor contact process satisfies the following property: Starting from a deterministic configuration $\eta_{0}$ and conditioning on the event $\eta_{t}(0)=1$, the collection of random variables $\left\{1-\eta_{t}(x): x<0\right\} \cup\left\{\eta_{t}(x): x>0\right\}$ is positively associated. Finally, in the last two sections, we discuss extensions to other models and derive some consequences and applications of the theorem and its corollaries following the ideas of [5].

\section{Proof of Theorem 1}

In this section we give a proof of Theorem 1, based on a Markov Chain introduced in [3]. Without loss of generality, we assume that $m=0$ and we let $A$ and $B$ be finite subsets of $L_{0}$ and $L_{n}$ respectively, such that $\Gamma_{\Lambda^{\prime}}(A, B) \neq \emptyset$. Then, we let $E$ be a finite set of oriented edges in $\Lambda^{\prime}$ containing at least one path from $A$ to $B$ and we let $S=\{0,1\}^{E}$. Each element $\eta$ of $S$ determines the state of the edges in $E$ in the natural way: $e \in E$ is open (closed) for $\eta$ if $\eta(e)=1(\eta(e)=0)$. Now, we only consider paths whose edges are in $E$ and we let $T$ be the subset of $S$ consisting of the elements for which there is an open path from $A$ to $B$. For $\eta \in T$, we let $\Gamma_{\ell}(\eta)\left(\Gamma_{r}(\eta)\right)$ be the leftmost (rightmost) open path from $A$ to $B$ under configuration $\eta$. We let $\sigma(S)$ be the $\sigma$-algebra of all subsets of $S$ and we let $P_{p}$ be the product probability measure on $\sigma(S)$ whose marginals are Bernoulli with parameter $p$. On the probability space $\left(S, \sigma(S), P_{p}\right)$, we define for each $e \in E$ a random variable $X_{e}$ by means of $X_{e}(\eta)=\eta(e)$.

Given a path $\gamma$ from $A$ to $B$ whose edges are in $E$, we let $\sigma_{r}(\gamma)$ be the $\sigma$-algebra generated by $\left\{X_{e}: e\right.$ is to the right of $\left.\gamma\right\}$ and we let $\sigma_{r}^{\prime}(\gamma)$ be the $\sigma$-algebra generated by $\left\{X_{e}: e\right.$ is strictly to the right of $\left.\gamma\right\}$. Similarly we let $\sigma_{\ell}(\gamma)$ be the $\sigma$-algebra generated by $\left\{X_{e}: e\right.$ is to the left of $\left.\gamma\right\}$ and we let $\sigma_{\ell}^{\prime}(\gamma)$ be the $\sigma$-algebra generated by $\left\{X_{e}: e\right.$ is strictly to the left of $\left.\gamma\right\}$. We now note that the event $\left\{\Gamma_{\ell}=\gamma\right\}$ is $\sigma_{\ell}(\gamma)$ measurable. Since the $\sigma$-algebras $\sigma_{\ell}(\gamma)$ and $\sigma_{r}^{\prime}(\gamma)$ are independent, this implies that 
under the conditional measure $P_{p}\left(\bullet \mid \Gamma_{\ell}=\gamma\right)$ the distribution of the state of the bonds which are strictly to the right of $\gamma$ remains a Bernoulli product measure of parameter $p$. Similarly, under the conditional measure $P_{p}\left(\bullet \mid \Gamma_{r}=\gamma\right)$ the distribution of the state of the bonds which are strictly to the left of $\gamma$ remains a Bernoulli product measure of parameter $p$. We now define a Markov Chain on T. Its transition mechanism is given in two steps. For a given initial state $\eta_{0} \in T$, first we choose $\eta_{1 / 2} \in T$ by letting $\eta_{1 / 2}(e)=\eta_{0}(e)$ for all $e$ to the left of $\Gamma_{\ell}\left(\eta_{0}\right)$ (this includes the edges on $\Gamma_{\ell}\left(\eta_{0}\right)$ ) and for the other elements of $E$ we let $\eta_{1 / 2}(e)$ be independent Bernoulli random variables with parameter $p$. Once we have determined $\eta_{1 / 2}$ we let $\eta_{1}(e)=\eta_{1 / 2}(e)$ for all $e$ to the right of $\Gamma_{r}\left(\eta_{1 / 2}\right)$ ( this includes the edges on $\Gamma_{r}\left(\eta_{1 / 2}\right)$ ) and for the other elements of $E$ we let $\eta_{1}(e)$ be independent Bernoulli random variables with parameter $p$ which are also independent of the random variables used to determine $\eta_{1 / 2}$. In the sequel we will need to consider this Markov Chain for different sets $E$. We will call it the Markov Chain associated to $E$. Besides this, we extend the notation of the previous sections by letting $\Gamma_{E}(A, B)$ be the set of paths from $A$ to $B$ whose edges are in $E$.

Proposition 1. (van den Berg, Häggström, Kahn) The measure $P_{p}(\bullet \mid T)$ is invariant for the Markov chain.

Proof. We show that if the initial state of the chain $\eta_{0}$ is chosen according to the distribution $P_{p}(\bullet \mid T)$, then $\eta_{1 / 2}$ has the same distribution. A similar argument will then show that $\eta_{1}$ has the same distribution as $\eta_{1 / 2}$. Let $\gamma$ be an arbitrary path in $\Gamma_{E}(A, B)$ and let

$$
S_{\gamma}=\left\{\eta \in T: \Gamma_{\ell}(\eta)=\gamma\right\}
$$

Then $\left(S_{\gamma}: \gamma \in \Gamma_{E}(A, B)\right)$ is a partition of $T$ and $P_{p}(\bullet \mid T)$ is a convex combination of the measures $\left(P_{p}\left(\bullet \mid S_{\gamma}\right)\right)_{\gamma \in \Gamma_{E}(A, B)}$. Therefore, it suffices to show that if $\eta_{0}$ is distributed according to some $P_{p}\left(\bullet \mid S_{\gamma}\right)$ then $\eta_{1 / 2}$ is also distributed according to that measure. But this is an immediate consequence of the way we obtain $\eta_{1 / 2}$ from $\eta_{0}$ and the already observed fact that the under the conditional measure $P_{p}\left(\bullet \mid \Gamma_{\ell}=\gamma\right)$ the distribution of the state of the bonds which are strictly to the right of $\gamma$ remains a Bernoulli product measure of parameter $p$.

Since the Markov Chain associated to $E$ is obviously irreducible and aperiodic we 
deduce from this proposition the following:

Corollary 3. From any initial distribution, the Markov Chain associated to E converges to $P_{p}(\bullet \mid T)$.

Proof of Theorem 1. We only prove the first inequality, since the other proofs are similar. Let $E$ be the set of edges belonging to paths in $\Gamma_{\Lambda^{\prime}}(A, B)$ and let $E_{\ell(G)}$ be the set of edges belonging to paths in $\Gamma_{\Lambda^{\prime} \cap \ell(G)}(A, B)$. We let $T_{1}$ be the subset of elements of $\{0,1\}^{E}$ for which there exists an open path from $A$ to $B$ and we let $T_{2}$ be the subset of elements of $\{0,1\}^{E_{\ell(G)}}$ for which there exists an open path from $A$ to $B$. We now construct a Markov Chain in

$$
X=\left\{(\eta, \xi) \in T_{1} \times T_{2}: \Gamma_{\ell}(\eta) \geq \Gamma_{\ell}(\xi), \Gamma_{r}(\eta) \geq \Gamma_{r}(\xi)\right\}
$$

whose first and second marginals are as the Markov Chains associated to $E$ and to $E_{\ell(G)}$ respectively. This is done as follows: Assume $\left(\eta_{0}, \xi_{0}\right) \in X$, then let $\left\{Y_{e}\right.$ : $e$ strictly to the right of $\left.\Gamma_{\ell}(\xi)\right\}$ be a collection of i.i.d Bernoulli random variables of parameter $p$. First note that by definition of $X, \Gamma_{\ell}(\eta) \geq \Gamma_{\ell}(\xi)$, then define $\eta_{1 / 2}$ and $\xi_{1 / 2}$ as follows:

$\eta_{1 / 2}(e)=\eta_{0}(e)$ for all $e$ to the left of $\Gamma_{\ell}\left(\eta_{0}\right)$,

$\eta_{1 / 2}(e)=Y_{e}$ for all $e$ strictly to the right of $\Gamma_{\ell}\left(\eta_{0}\right)$,

$\xi_{1 / 2}(e)=\xi_{0}(e)$ for all $e$ to the left of $\Gamma_{\ell}\left(\xi_{0}\right)$,

$\xi_{1 / 2}(e)=Y_{e}$ for all $e$ strictly to the right of $\Gamma_{\ell}\left(\xi_{0}\right)$.

After that note that $\left(\eta_{1 / 2}, \xi_{1 / 2}\right) \in X$ and let $\left\{Z_{e}: e\right.$ strictly to the left of $\left.\Gamma_{r}(\eta)\right\}$ be collection of i.i.d Bernoulli random variables of parameter $p$ which is independent of the random variables $Y_{e}$. Finally, define $\eta_{1}$ and $\xi_{1}$ as follows:

$\eta_{1}(e)=\eta_{1 / 2}(e)$ for all $e$ to the right of $\Gamma_{r}\left(\eta_{1 / 2}\right)$,

$\eta_{1}(e)=Z_{e}$ for all $e$ strictly to the left of $\Gamma_{r}\left(\eta_{1 / 2}\right)$,

$\xi_{1}(e)=\xi_{1 / 2}(e)$ for all $e$ to the right of $\Gamma_{r}\left(\xi_{1 / 2}\right)$,

$\xi_{1}(e)=Z_{e}$ for all $e$ strictly to the left of $\Gamma_{r}\left(\xi_{1 / 2}\right)$.

We can now complete our proof: let $\Phi$ be an increasing function on $\Gamma_{E}(A, B)$ and let $\left(\eta_{0}, \xi_{0}\right)$ be an element of $X$. Then $\left(\eta_{n}, \xi_{n}\right) \in X$ a.s. $\forall n$. Therefore,

$$
\Phi\left(\Gamma_{\ell}\left(\eta_{n}\right)\right) \geq \Phi\left(\Gamma_{\ell}\left(\xi_{n}\right)\right) a . s . \quad \forall n .
$$


Hence,

$$
E\left(\Phi\left(\Gamma_{\ell}\left(\eta_{n}\right)\right)\right) \geq E\left(\Phi\left(\gamma_{\ell}\left(\xi_{n}\right)\right)\right) \forall n
$$

Now, taking limits as $n$ goes to infinity and applying Corollary 3 to both sides of the inequality we get $\mu_{\Lambda^{\prime}}(A, B) \geq \mu_{\Lambda^{\prime} \cap \ell(G)}(A, B)$.

\section{Generalizations and extensions of Theorem 1}

We first discuss generalizations of the oriented bond percolation model that do not require any change in our proof of the main results, then we consider oriented site percolation and the contact process and after that we mention an extension of these results which require a quite different proof.

It is easy to check that we never made any use of the assumption that all of the bonds have the same probability of being open. In fact, we could assign a different probability to each bond, and the proofs will continue to work without any changes. It may seem that this is an uninteresting generalization, but we will see that it turns out to be relevant when we use percolation models to approximate the continuous time contact process.

We now turn to oriented site percolation. Let

$$
\bar{\Lambda}=\{(x, y): x, y \in \mathbb{Z}, y \geq 0\}
$$

Fix integers $a \leq 0<b$, and for each $(x, y) \in \bar{\Lambda}$, introduce oriented bonds from $(x, y)$ to $(x+k, y+1)$ for $a \leq k \leq b$. All of the bonds are open. The sites are open independently of each other, with probability $p \in(0,1)$ (we could also allow different probabilities for different sites). Paths are defined in the obvious way, and open paths are paths in which all of the sites are open.

The case where $a=0$ and $b=1$ is equivalent to the standard oriented site percolation in $\mathbb{Z}^{2}$, where there are two bonds per site. Not surprisingly, our proofs of the main result can be modified in a routine way to cover this case.

Once the case $a=0, b=1$ is handled, it is quite routine to further modify the proof to cover arbitrary $a \leq 0<b$, which is to say that our main result holds for finite range oriented site percolation in 2 dimensions. This highlights a significant difference between oriented bond percolation and oriented site percolation. In oriented bond 
percolation, the leftmost and rightmost paths may not even exist when there is the possibility that bonds cross one another, as will be the case when $b-a>1$. But in oriented site percolation, leftmost and rightmost paths always exist, for any choice of $a, b$.

Finally, we briefly discuss the contact process. By treating the $y$-coordinate in $\Lambda$ as the time variable, one can obtain various versions of the discrete time contact process from oriented percolation. The standard model is equivalent to oriented site percolation. Variations on this model can be obtained from oriented bond percolation, and also by looking at mixed models in which both the sites and the bonds can be open or closed. Not surprisingly, our main results applies to many mixed percolation models, and hence to many different discrete time contact processes.

One way to extend our results to the continuous time contact process is to approximate continuous time with discrete time. This method works easiest for the one-sided nearest neighbor contact process. Then we could use the oriented bond percolation model that is the setting for most of this paper, but it is perhaps more natural to do oriented bond percolation on an equivalent graph: the set of sites is $\bar{\Lambda}$ and the oriented bonds are those that correspond to $a=0$ and $b=1$. That is, two oriented bonds emerge from each site $(x, y)$, a "vertical" bond connecting it to the site $(x, y+1)$ and a "contact" bond connecting it to $(x+1, y+1)$.

With this setup, we can approximate the continuous time one-sided contact process by letting the contact bonds be open with small probability $h>0$ and letting the vertical bonds be open with probability $1-\varepsilon h$, where $\varepsilon \geq 0$ is a parameter of the model. Then letting $h \downarrow 0$ and rescaling time by a factor of $h$ produces the continuoustime model. This shows why it can be desirable to allow the bonds to have different probabilities of being open.

One can use a similar approximation method for the two-sided nearest neighbor contact process. In this case, we use the graph $\Lambda$ that is the setting for the bulk of this paper, but we add additional oriented "vertical bonds" that connect each site $(x, y) \in \Lambda$ to the site $(x, y+2)$. Then by assigning appropriate probabilities to the bonds (different for the vertical bonds than for the diagonal bonds), one obtains a percolation model that depends on a parameter $h$, and this model converges to the two-sided nearest neighbor contact process as $h \rightarrow 0$. See [2] for further details. The 
bottom line is that the results in this paper all apply to one the one-sided and two-sided contact processes in continuous time.

Finally, the assumption of independence between bonds may be weakened. Keeping the independence for bonds emerging from different sites but allowing the two bonds emerging from the same site to be dependent, our main results still hold. But when the bonds emerging from the same site are negatively correlated, Theorem 1 requires a completely different proof. This proof uses a rather intricate inductive argument, that was hinted at in [5], rather than the Markov Chain of Section 2. For details of this proof we refer the reader to [1].

\section{Applications of Theorem 1}

One reason for our interest in extreme paths is that they provide us with a useful way to analyze various conditional probabilities, and with the help of Theorem 1, we are able to make comparisons that go beyond the usual correlation inequalities that are familiar in percolation theory. The results in this section apply to the more general models discussed in the previous section, except that Corollaries 5, 6, 7 and 8 require translation invariance, so that all of the bond (or site) probabilities must be the same.

In this section we adopt the following notations: Given $0 \leq m \leq n$, a fixed subset $\Lambda^{\prime}$ of $\Lambda$ and finite subsets $A$ and $B$ of $L_{m}$ and $L_{n}$ respectively, we let

$$
H=\left\{\text { there exists an opent path from } A \text { to } B \text { in } \Lambda^{\prime}\right\} .
$$

Then, if $F$ is another event such that $P(F \cap H)>0$, we let $\mu_{\Lambda^{\prime}}^{F}(A, B)$ be the distribution of the leftmost open path from $A$ to $B$ in $\Lambda^{\prime}$ given the event $F \cap H$. The same notation will apply to the distributions of rightmost paths where $\nu$ will substitute for $\mu$.

We will rely on a key fact about extreme paths. It is that if $\gamma$ is a path, the event that $\gamma$ is a rightmost (leftmost) open path is measurable with respect to the states of the bonds that are to the right (left) of $\gamma$ and hence this event is independent of the states of the bonds that are strictly to the left (right) of $\gamma$.

The following result and its proof show how we use this fact in conjunction with Theorem 1 to compare several different conditional probabilities.

Lemma 1. Let $n>0$, let $A$ be a finite subset of $L_{0}$ and let $B_{1}, B_{2}, B_{3}$ be finite subsets 
of $L_{n}$. Suppose that $B_{1}$ is strictly to the left of $B_{2}$ and that $B_{2}$ is strictly to the left of $B_{3}$. For $i=1,2,3$, define the events

$$
H_{i}=\left\{\text { there exists an open path from } A \text { to } B_{i}\right\} .
$$

If the events $\mathrm{H}_{2}$ and $\mathrm{H}_{3}$ have nonzero probability, then

$$
P\left(H_{1} \mid H_{2} \cap H_{3}^{c}\right) \geq P\left(H_{1} \mid H_{2}\right) \geq P\left(H_{1} \mid H_{2} \cap H_{3}\right)
$$

and

$$
P\left(H_{1} \mid H_{2}\right) \geq P\left(H_{1} \mid H_{3}\right) .
$$

Furthermore, if $A$ consists of a single site $(x, 0)$, then $P\left(H_{1} \mid H_{2}\right)$ is nonincreasing in $x$ for all $x$ such that the event $\mathrm{H}_{2}$ has positive probability.

Proof. We begin by proving the following inequality:

$$
\nu^{H_{3}^{c}}\left(A, B_{2}\right) \leq \nu\left(A, B_{2}\right) .
$$

The event $H_{3}^{c}$ is the disjoint union of events of the form $\{\Phi=\varphi\}$, where $\Phi$ is the random set of all edges that are contained in open paths that end in $B_{3}$. The left side of (3) is a convex combination of the measures $\nu^{\{\Phi=\varphi\}}\left(A, B_{2}\right)$, and for each $\varphi$, $\nu^{\{\Phi=\varphi\}}\left(A, B_{2}\right)=\nu_{\ell(G)}\left(A, B_{2}\right)$, where $G$ is the set that contains the endpoints of the edges in $\varphi$. The inequality in (3) now follows from Theorem 1.

We now use (3) to prove the first inequality in (1). Let $\Gamma$ be the rightmost open path from $A$ to $B_{2}$, assuming that such a path exists, which is the same as assuming that $H_{2}$ occurs. The left side of (3) is the conditional distribution of $\Gamma$ given $H_{2} \cap H_{3}^{c}$ and the right side of (3) is the conditional distribution of $\Gamma$ given $H_{2}$. In either case, the event $H_{1}$ occurs if and only if there is an open path from $A \cup \Gamma$ to $B_{1}$.

Let $\gamma$ be any path from $A$ to $B_{2}$. Given the event $H_{2} \cap\{\Gamma=\gamma\}$, the edges strictly to the left of $\gamma$ are each open with probability $p$ and they are independent of each other. This statement about the edges left of $\gamma$ also holds true given the event $H_{2} \cap H_{3}^{c} \cap\{\Gamma=$ $\gamma$ \} because of the assumption that $B_{3}$ is strictly to the right of $B_{2}$. In either case, whether or not there is an open path from $A \cup \gamma$ to $B_{1}$ is determined in the same way by the openness of the edges that are strictly to the left of $\gamma$. Thus, there is a function $\varphi$ on the set of all paths $\gamma$ from $A$ to $B_{2}$ such that

$$
\varphi(\gamma)=P\left(H_{1} \mid H_{2} \cap\{\Gamma=\gamma\}\right)=P\left(H_{1} \mid H_{2} \cap H_{3}^{c} \cap\{\Gamma=\gamma\}\right),
$$


and we have

$$
E\left(\varphi(\Gamma) \mid H_{2}\right)=P\left(H_{1} \mid H_{2}\right) \quad \text { and } \quad E\left(\varphi(\Gamma) \mid H_{2} \cap H_{3}^{c}\right)=P\left(H_{1} \mid H_{2} \cap H_{3}^{c}\right)
$$

Assume $\gamma_{1}$ and $\gamma_{2}$ are possible values of $\Gamma$ such that $\gamma_{1} \leq \gamma_{2}$. Then, for $i=1,2$ we have

$$
P\left(H_{1} \mid H_{2} \cap\left\{\Gamma=\gamma_{i}\right\}\right)=P\left(A \cup \gamma_{i} \rightarrow B_{1}\right),
$$

where $\left\{A \cup \gamma_{i} \rightarrow B_{1}\right\}$ is the event occurring iff there is an open path from $A \cup \gamma_{i}$ to $B_{1}$. Since

$$
\left\{A \cup \gamma_{2} \rightarrow B_{1}\right\} \subset\left\{A \cup \gamma_{1} \rightarrow B_{1}\right\}
$$

the function $\varphi(\gamma)$ is decreasing with respect to the partial ordering on paths, so the first inequality in (1) now follows from (3). The second inequality in (1) follows immediately from the first inequality and the fact that the middle expression in (3) is a convex combination of the first and third expressions.

To prove (2), we first use Corollary 2 to get:

$$
\nu\left(A, B_{2}\right) \leq \nu\left(A, B_{3}\right) .
$$

Now (2) follows, in the same way that (1) followed from (3).

To prove the last part of the lemma, we note that by Corollary 2 ,

$$
\nu\left((x, 0), B_{2}\right) \leq \nu\left((x+2,0), B_{2}\right)
$$

for all $x$ such that there exist paths from $(x, 0)$ and $(x+2,0)$ to $B_{2}$. The last part of the lemma is now proved in the same way that (1) and (2) were proved.

The first inequality in (1) may seem counterintuitive. We know that the occurence of a "negative" event like $H_{3}^{c}$ makes a "positive" event like $H_{1}$ less likely to occur. But the first inequality says informally that once $\mathrm{H}_{2}$ occurs, the additional occurrence of the negative event $H_{3}^{c}$ makes $H_{1}$ more likely to occur. Here is another way to state this result:

Corollary 4. Let $H_{1}, H_{2}, H_{3}$ be as in Lemma 1. Then given $H_{2}$, the events $H_{1}$ and $\mathrm{H}_{3}$ are conditionally negatively correlated. 
Proof. The proof is elementary, using the second inequality in Lemma 1:

$$
\begin{gathered}
P\left(H_{1} \cap H_{3} \mid H_{2}\right)=\frac{P\left(H_{1} \cap H_{2} \cap H_{3}\right)}{P\left(H_{2}\right)}=\frac{P\left(H_{1} \cap H_{2} \cap H_{3}\right)}{P\left(H_{2} \cap H_{3}\right)} P\left(H_{3} \mid H_{2}\right) \\
=P\left(H_{1} \mid H_{2} \cap H_{3}\right) P\left(H_{3} \mid H_{2}\right) \leq P\left(H_{1} \mid H_{2}\right) P\left(H_{3} \mid H_{2}\right) .
\end{gathered}
$$

Here is another application of Lemma 1. It is a rather natural monotonicity involving certain percolation probabilities. It is somewhat surprising that its proof seems to require the consequences of something as sophisticated as Theorem 1. We note that this result clearly depends on some translation invariance, so it requires all of the bond probabilities (or site probabilities in the case of oriented site percolation) to be the same. A different proof of this result is given in [2], but we believe the one given here is more natural and easier to follow.

Corollary 5. Let $0 \leq m<n$ and let $x, y$ be integers such that $(x, m) \in L_{m}$ and $(y, n) \in L_{n}$. Let $A_{x, y}$ be the event that there is an open path from $(x, m)$ to $(y, n)$. Then $P\left(A_{x, y}\right)$ is nonincreasing in $|x-y|$.

Proof. Because of the natural symmetries built into the percolation model, we may assume without loss of generality that $m=0, x=0$ and $y \geq 0$. The obvious inductive argument reduces the proof to showing that

$$
P\left(A_{0, y}\right) \geq P\left(A_{0, y+2}\right),
$$

where we may assume that $y$ is such that there exists at least one path from $(0,0)$ to $(n, y+2)$. Since $y \geq 0$, this assumption implies that there also exists at least one path from $(0,0)$ to $(n, y)$.

Under these circumstances, to prove (4), it is enough to prove that

$$
P\left(A_{0, y} \mid A_{0, y+2}\right) \geq P\left(A_{0, y+2} \mid A_{0, y}\right)
$$

since the numerators in the expressions for the two conditional probabilities in (5) are the same and since the denominators in these expressions are the two sides of (4) (in reverse order).

To prove (5), we first use left-right symmetry and then translation invariance to get

$$
P\left(A_{0, y+2} \mid A_{0, y}\right)=P\left(A_{0,-y-2} \mid A_{0,-y}\right)=P\left(A_{2 y+2, y} \mid A_{2 y+2, y+2}\right) .
$$


Since we assumed that $y \geq 0$, the last part of Lemma 1 gives us

$$
P\left(A_{2 y+2, y} \mid A_{2 y+2, y+2}\right) \leq P\left(A_{0, y} \mid A_{0, y+2}\right)
$$

proving (5), and thus (4). Note that our assumptions about $y$ ensure that all of the relevant events in these applications of Lemma 1 have positive probability, as required by the hypotheses of that lemma.

For our last results we adopt the following notation: For $(k, n) \in \Lambda$ we let $X(k, n)$ be the indicator function of the event $\{(0,0) \rightarrow(k, n)\}$, and if $\gamma$ is a path in $\Lambda$ we let $X^{\gamma}(k, n)$ be the indicator function of the event $\{\gamma \rightarrow(k, n)\}$.

Corollary 6. Let $y \in \mathbb{Z}$ and $n \in \mathbb{N}$ be such that $(y, n) \in \Lambda$ and $-n \leq y \leq n-2$. Then for any $k \in \mathbb{N}$ and any increasing function $f:\{0,1\}^{k} \rightarrow \mathbb{R}$, we have

$$
\begin{gathered}
E(f(X(y+2, n), \ldots, X(y+2 k, n)) \mid X(y, n)=1) \geq \\
E(f(X(y+4, n), \ldots, X(y+2 k+2, n)) \mid X(y+2, n)=1) .
\end{gathered}
$$

Proof. On the event $\{X(y, n)=1\}$ we let $\Gamma$ be the leftmost open path from $(0,0)$ to $(y, n)$. Then,

$$
\begin{gathered}
E(f(X(y+2, n), \ldots, X(y+2 k, n)) \mid X(y, n)=1)= \\
\int_{\gamma} E(f(X(y+2, n), \ldots, X(y+2 k, n)) \mid \Gamma=\gamma) d \mu((0,0),(y, n))(\gamma)= \\
\int_{\gamma} E\left(f\left(X^{\gamma}(y+2, n), \ldots, X^{\gamma}(y+2 k, n)\right)\right) d \mu((0,0),(y, n))(\gamma),
\end{gathered}
$$

where for the second equality we used the fact that conditioning on the event $\{\Gamma=\gamma\}$ does not change the distribution of the state of the bonds strictly to the right of $\gamma$. Similarly,

$$
\begin{gathered}
E(f(X(y+4, n), \ldots, X(y+2 k+2, n)) \mid X(y+2, n)=1)= \\
\int_{\gamma} E(f(X(y+4, n), \ldots, X(y+2 k+2, n)) \mid \Gamma=\gamma) d \mu((0,0),(y+2, n))(\gamma)= \\
\int_{\gamma} E\left(f\left(X^{\gamma}(y+4, n), \ldots, X^{\gamma}(y+2 k+2, n)\right)\right) d \mu((0,0),(y+2, n))(\gamma)= \\
\int_{\gamma} E\left(f\left(X^{\gamma}(y+2, n), \ldots, X^{\gamma}(y+2 k, n)\right)\right) d \mu((-2,0),(y, n))(\gamma),
\end{gathered}
$$

where the last equality follows from translation invariance. But by the first inequality of Corollary $2, \mu((-2,0),(y, n)) \leq \mu((0,0),(y, n))$, and the corollary follows from the 
fact that

$$
F(\gamma)=E\left(f\left(X^{\gamma}(y+2, n), \ldots, X^{\gamma}(y+2 k, n)\right)\right)
$$

is an increasing function on the set $\Gamma(\{(-2,0),(0,0)\},\{(y, n)\})$.

Corollary 7. Let $x \in \mathbb{N}_{0}$ and $n \in \mathbb{N}$ be such that $(x, n) \in \Lambda$ and let $a_{1}, \ldots, a_{k}$ be an increasing sequence of elements of $2 \mathbb{N}_{0}$. Then,

$$
\begin{gathered}
P\left(X\left(x+a_{1}, n\right)=1, \ldots, X\left(x+a_{k}, n\right)=1\right) \geq \\
P\left(X\left(x+a_{1}+2, n\right)=1, \ldots, X\left(x+a_{k}+2, n\right)=1\right) .
\end{gathered}
$$

Proof. Write

$$
\begin{gathered}
P\left(X\left(x+a_{1}, n\right)=1, \ldots, X\left(x+a_{k}, n\right)=1\right)= \\
P\left(X\left(x+a_{2}, n\right)=1, \ldots, X\left(x+a_{k}, n\right)=1 \mid X\left(x+a_{1}, n\right)=1\right) \\
P\left(X\left(x+a_{1}, n\right)=1\right)
\end{gathered}
$$

and

$$
\begin{gathered}
P\left(X\left(x+a_{1}+2, n\right)=1, \ldots, X\left(x+a_{k}+2, n\right)=1\right)= \\
P\left(X\left(x+a_{2}+2, n\right)=1, \ldots, X\left(x+a_{k}+2, n\right)=1 \mid X\left(x+a_{1}+2, n\right)=1\right) \\
P\left(X\left(x+a_{1}+2, n\right)=1\right) .
\end{gathered}
$$

Now we only need to show that the right hand side of (8) is greater than or equal to the right hand side of (9), but this follows immediately from Corollaries 5 and 6 .

Remark: Of course, unlike Corollary 6 , this last result is false if we do not assume that $x \geq 0$. It falls short of proving that under the same hypothesis the random vector $\left(X\left(x+a_{1}, n\right), \ldots, X\left(x+a_{k}, n\right)\right)$ is stochastically above $\left(X\left(x+a_{1}+2, n\right), \ldots, X(x+\right.$ $\left.\left.a_{k}+2, n\right)\right)$ but we conjecture that this is also true.

Our last application generalizes Corollary 5.

Corollary 8. Let $0 \leq m<n$, let $k \in \mathbb{N}$ and let $x, y$ be integers such that $(x, m) \in L_{m}$ and $(y, n) \in L_{n}$. Let $A_{x, y, k}$ be the event that there is an open path from $(x, m)$ to $\{(y-2 k, n), \ldots,(y+2 k, n)\}$. Then $P\left(A_{x, y, k}\right)$ is nonincreasing in $|x-y|$. 
Proof. As in the proof of Corollary 5, we may assume without loss of generality that $m=0, x=0$ and $y \geq 0$ and an obvious inductive argument reduces the proof to showing that

$$
P\left(A_{0, y, k}\right) \geq P\left(A_{0, y+2, k}\right)
$$

This inequality is trivial if there are no paths from $(0,0)$ to $(y+2 k+2, n)$. Hence, we may assume that

$$
|y+2 k+2| \leq n
$$

Now, (10) will follow from

$$
P\left(A_{0, y, k} \cap A_{0, y+2, k}^{c}\right) \geq P\left(A_{0, y, k}^{c} \cap A_{0, y+2, k}\right) .
$$

This last inequality is equivalent to:

$$
\begin{array}{r}
P(X(y-2 k, n)=1, X(y-2 k+2, n)=0, \ldots, X(y+2 k+2, n)=0) \geq \\
P(X(y+2+2 k, n)=1, X(y+2 k, n)=0, \ldots, X(y-2 k, n)=0) .
\end{array}
$$

The left and right hand sides of this inequality can be written respectively as

$$
\begin{gathered}
P(X(y-2 k+2, n)=0, \ldots, X(y+2 k+2, n)=0 \mid X(y-2 k, n)=1) \\
P(X(y-2 k, n)=1)
\end{gathered}
$$

and

$$
\begin{gathered}
P(X(y+2 k, n)=0, \ldots, X(y-2 k, n)=0 \mid X(y+2+2 k, n)=1) \\
P(X(y+2+2 k, n)=1) .
\end{gathered}
$$

Hence, the corollary will follow from:

$$
P(X(y-2 k, n)=1) \geq P(X(y+2+2 k, n)=1)
$$

and

$$
\begin{gathered}
P(X(y-2 k+2, n)=0, \ldots, X(y+2 k+2, n)=0 \mid X(y-2 k, n)=1) \geq \\
P(X(y+2 k, n)=0, \ldots, X(y-2 k, n)=0 \mid X(y+2+2 k, n)=1) .
\end{gathered}
$$


Now, (16) follows from Corollary 5 and our assumption on $y$, hence it remains to prove (17). To do so, first note that by symmetry the right hand side of (17) is equal to

$$
P(X(-y-2 k, n)=0, \ldots, X(-y+2 k, n)=0 \mid X(-y-2-2 k, n)=1) .
$$

Proceeding as in proof of Corollary 7 and recalling (11) we see that this is equal to

$$
\int_{\gamma} P\left(X^{\gamma}(-y-2 k, n)=0, \ldots, X^{\gamma}(-y+2 k, n)=0\right) d \mu((0,0),(-y-2-2 k, n))(\gamma),
$$

which by translation invariance is equal to

$$
\int_{\gamma} P\left(X^{\gamma}(y-2 k+2, n)=0, \ldots, X^{\gamma}(y+2 k+2, n)=0\right) d \mu((2 y+2,0),(y-2 k, n))(\gamma) .
$$

Since the left hand side of (17) is equal to

$$
\int_{\gamma} P\left(X^{\gamma}(y-2 k+2, n)=0, \ldots, X^{\gamma}(y+2 k+2, n)=0\right) d \mu((0,0),(y-2 k, n))(\gamma),
$$

the result follows from Corollary 2, our assumption on $y$ and the fact that

$$
P\left(X^{\gamma}(y-2 k+2, n)=0, \ldots, X^{\gamma}(y+2 k+2, n)=0\right)
$$

is a decreasing function of $\gamma$ on the set $\Gamma(\{(0,0),(2 y+2,0)\},\{(y-2 k, n)\})$.

Remark: We did not find a way to complete the proof of Theorem 5 in [5] which remains as an open problem.

Acknowledgments: We thank the referee's careful reading and helpful comments. This paper was written while the first author was visiting IMPA and was supported first by Capes and then by Cnpq.

\section{References}

[1] E.D. Andjel, L.F. Gray (2014) Extreme paths in oriented 2D percolation. http://arxiv.org/abs/1411.0956

[2] E. Andjel, M. Sued (2008). An inequality for oriented 2-D percolation. In In and out of equilibrium II. (M.E. Vares and V. Sidoravicius, eds). Progress in Probability 60, 21-30. Birkhauser Verlag, Basel.

[3] J. van den Berg, O. Häggström. J. Kahn (2006). Proof of a conjecture of N. Konno for $1 \mathrm{D}$ contact process. IMS Lecture Notes, Dynamics 83 Stochastics 48, 16-23. 
[4] R. Durrett (1984). Oriented percolation in two dimensions. Ann. Probab. 12, 999-1040.

[5] L.F. Gray. (1991) Is the contact process dead? Lectures in Appli. Math. 27, 19-29. 